\title{
Introduction to the special section on designing automated and connected driving systems to work with people
}

\author{
Klaus Bengler ${ }^{1}$. Oliver Carsten ${ }^{2}$
}

Published online: 13 February 2019

(c) Springer-Verlag London Ltd., part of Springer Nature 2019

Automated road vehicles, which were for a long time only a dream, are now, with rapid advances in sensing systems and computing power, becoming a reality. Lower levels of automated driving, providing automated control within a lane, are now commonly available on production vehicles, while higher levels of automation, that no longer require human attention to the roadway, are under test and promised for production vehicles in the next few years.

The introduction of automated driving has to be understood as a remarkable paradigm shift, as remarkable as the introduction of the "automobile" into mass use in the first half of the twentieth century. Other technology domains such as aviation serve in many discussions as an example. But the introduction of automation into driving raises more and different questions than the autopilot for airplanes. The potential for fully autonomous vehicles raises additional questions concerning human usage and interaction.

While this technological development is ongoing, relevant questions have to be answered to ensure safe and ergonomic designs that take human factors aspects into account. Here both methodological procedures as well as conceptual guidelines are important. These can be based on existing knowledge, but specific additional studies are needed as well, especially since there is limited experience on how humans interact with automated vehicles both internally (from a driver or passenger perspective) and externally (from the perspective of other road users).

The contribution of Carsten and Martens focusses especially on basic human factors recommendations and

Oliver Carsten

O.M.J.Carsten@its.leeds.ac.uk

Klaus Bengler

bengler@tum.de

1 Lehrstuhl für Ergonomie, Technische Universität München, Boltzmannstraße 15, 85747 Garching, Germany

2 Institute for Transport Studies (ITS), University of Leeds, 34-40 University Road, Leeds LS2 9JT, UK principles that should be taken into account when implementing automation in a road vehicle.

The study of Stoll et al. describes the complexity of lane change manoeuvres and potential strategies to implement cooperation between automated vehicles and manually driven cars.

Feldhüttter et al. give insight into the effects of longer automation periods and their effect on driver status. The study also provides information on how to conduct longduration automation experiments and on data sampling.

Trust and compliance play a vital role in human interaction with vehicle automation. As the automated system will not be perfect, technical reliability will influence users' trust and usage behaviour. Schwarz et al. present experimental results on these issues.

Solis-Marcos and Kircher focus on another central element of automation, namely workload. In particular they address the correspondence between subject measures of workload such as NASA-TLX and psychophysiological measures. Their findings suggest that Event Related Potentials (ERPs) provide complementary information to traditional mental workload measures.

Finally, Schieben et al. focus on the interaction of automated vehicles with external traffic participants. They propose a design framework to support the designers of automated vehicles designers in reducing the complexity of the design space, outlining four categories of information that are needed by humans interacting with automated vehicles.

Overall, there is a cautionary tale here. It took 70 or 80 years for society in the highly industrialised counties to seriously grapple with the negative consequences of humandriven road traffic, and even longer before those negative consequences became a concern at the world level. Although the development of automated driving is being driven forward with remarkable speed in research and industry, the human factors and safety aspects require concerted attention. Careful thought and experimental thoroughness are of 
the greatest importance. Only reliable and valid results can provide a good basis for industrial and political decisions.
Publisher's Note Springer Nature remains neutral with regard to jurisdictional claims in published maps and institutional affiliations. 